\title{
Diagnosis of retrotracheal left brachiocephalic vein with prenatal ultrasound: a case report
}

\author{
Yuan Zhao ${ }^{1 \wedge}$, Xueqin Ji ${ }^{1}$, Lili Ding ${ }^{2}$, Yan Xia ${ }^{1}$, Yang $\mathrm{Wu}^{1}$, Rui Xu ${ }^{1}$, Ning $\mathrm{Wu}^{1}$ \\ ${ }^{1}$ Department of Ultrasound, Ningxia Hui Autonomous Region Maternity and Child Health Hospital \& Ningxia Children's Hospital, Yinchuan, \\ China; ${ }^{2}$ Division of Obstetrical \& Gynecological Ultrasound, General Hospital of Ningxia Medical University, Yinchuan, China \\ Correspondence to: Xueqin Ji. Department of Ultrasound, Ningxia Hui Autonomous Region Maternity and Child Health Hospital \& Ningxia \\ Children's Hospital, No. 127, Hupan Road, Jinfeng District, Yinchuan 750011, Ningxia Hui Autonomous Region, China. Email: jinian54@163.com.
}

\begin{abstract}
Retroesophageal or retrotracheal left brachiocephalic vein (LBCV) is a rare anatomic variant that is often associated with congenital heart disease. It is rarely reported in fetal life, and an isolated fetal retroesophageal LBCV has a good prognosis: it is typically asymptomatic, although respiratory symptoms or swallowing disorders occasionally occur. A variant was observed on fetal echocardiography at $22^{+6}$ weeks of gestation. The 3-vessel view revealed a transverse section of a vessel to the left of the pulmonary artery. Tracing upwards along its long axis showed that the left subclavian vein joined the left internal jugular vein to form the LBCV, and tracing downwards revealed that the vessel traveled to the right and lower side, where it merged into the superior vena cava via the azygos vein behind the aortic arch. The variant was identified as retrotracheal LBCV. Three-dimensional (3D) reconstruction of fetal great vessels was performed using temporal spatial correlation imaging. The left internal jugular vein and the left subclavian vein converged into the LBCV, then bypassed behind the trachea and converged into the superior vena cava via the azygous vein. As a 3D technique, spatio-temporal image correlation (STIC) can visualize the abnormal courses of LBCV, thus improving the diagnostic accuracy. This article presents the 2-dimensional (2D) ultrasound, color Doppler, and STIC findings of an isolated retrotracheal LBCV, which may inform the sonographic diagnosis of such variants.
\end{abstract}

Keywords: Left brachiocephalic vein (LBCV); trachea; prenatal echocardiography; case report

Submitted Mar 26, 2021. Accepted for publication Jul 06, 2021.

doi: $10.21037 /$ tp-21-230

View this article at: https://dx.doi.org/10.21037/tp-21-230

\section{Introduction}

The left brachiocephalic vein ( $\mathrm{LBCV}$ ), also known as the left innominate vein, is formed by the confluence of the left subclavian vein and the left internal jugular vein. It is longer than the right brachiocephalic vein and travels anterior to the left common carotid artery and the brachiocephalic trunk and posterior to the right first sternoclavicular joint. It joins with the right brachiocephalic vein to form the superior vena cava, which carries blood from the head and neck to the right atrium. Anomalous LBCV is rare and often associated with congenital heart disease and aortic arch anomalies. Here, we report an isolated retrotracheal $\mathrm{LBCV}$ variant identified during the fetal period. We present the following article in accordance with the CARE reporting checklist (available at https://dx.doi.org/10.21037/ tp-21-230).

\section{Case presentation}

A 20 -year-old $\mathrm{G}_{1} \mathrm{P}_{0}$ woman presented. She was previously healthy and denied any history of tuberculosis, diabetes mellitus, hypertension, or cardiovascular disease. In her first trimester, she regularly took folic acid supplements and had

\footnotetext{
$\wedge$ ORCID: 0000-0002-9551-0809.
} 


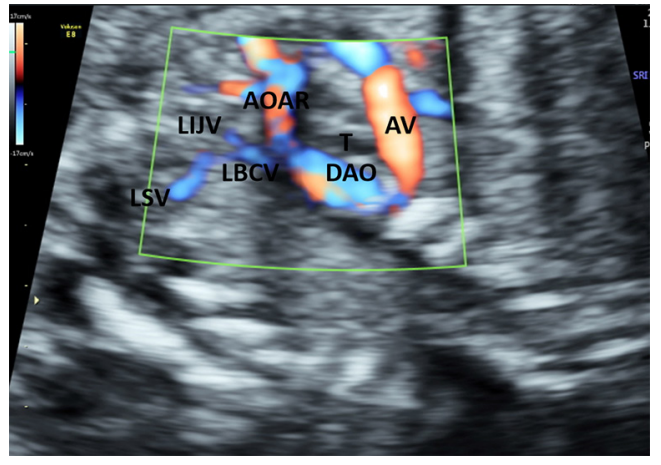

Figure 1 The oblique coronal view shows the LBCV to be formed by the confluence of the LSV and LIJV, traveling to the AV posterior to the trachea. LBCV, left brachiocephalic vein; LSV, left subclavian vein; LIJV, left internal jugular vein; AV, azygos vein.

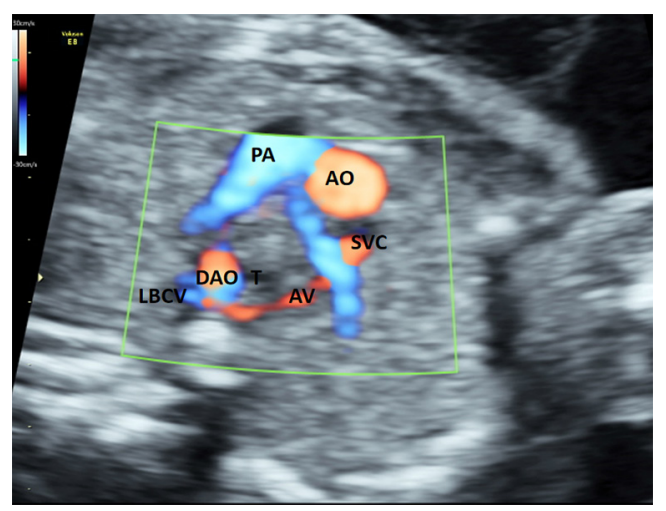

Figure 2 The 3 -vessel view shows the LBCV merging into the SVC via the AV posterior to the trachea. LBCV, left brachiocephalic vein; SVC, superior vena cava; AV, azygos vein.

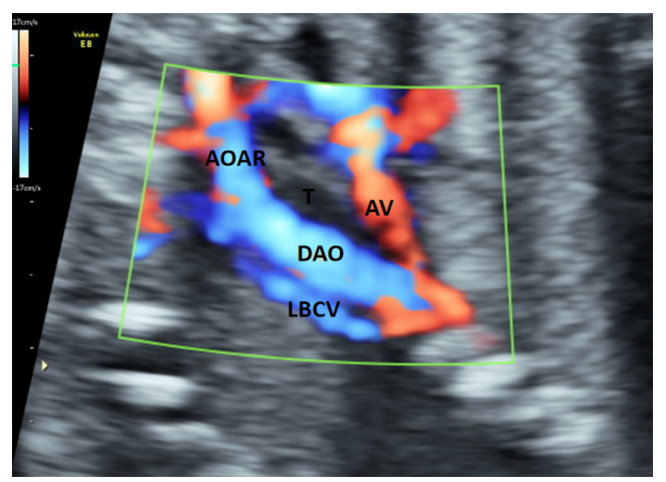

Figure 3 The oblique coronal view shows the $\mathrm{LBCV}$ traveling to the AV posterior to the DAO. LBCV, left brachiocephalic vein; AV, azygos vein; DAO, descending aorta. no vaginal bleeding, and screening for Down's syndrome screening showed a low risk. Ultrasound examination in the first trimester also showed no abnormality. During the echocardiography performed on gestational week $22^{+6}$, the 4-chamber view showed the following findings: the cardiothoracic ratio was approximately 0.34 (area ratio); the apex of the heart pointed to the left of the chest; ventricular dextro-loop was present; the left and right cardiac chambers were basically symmetrical; the atrioventricular connections were concordant; the opening and closing of the mitral and tricuspid valves were visible; a crisscross structure was found, with the valve of the foramen ovale in the interatrial septum drifted from the right to the left atrium. The right ventricular outflow tract (RVOT) view [or three-vessel view $(3 \mathrm{VV})$ ] showed that the aorta and pulmonary artery were crossed at their roots, with normal diameters; also, the opening and closing of the aortic and pulmonary valves were visible. The $3 \mathrm{VV}$ revealed normal diameters of the aortic arch and ductus arteriosus. A vascular structure was observed at the left of the pulmonary artery. Tracing upwards along its long axis showed that the left subclavian vein joined the left internal jugular vein to form the LBCV (Figure 1), and tracing downwards revealed that the vessel traveled to the right and lower side, where it merged into the superior vena cava via the azygos vein behind the aortic arch (Figures 2-5). The superior vena cava and inferior vena cava connected to the right atrium Ultrasound suggested the structure was a retrotracheal LBCV. The pregnancy was continued, and the diagnosis was confirmed after birth. As of writing, the infant is 10 months old, and there is no significant discomfort or other symptoms. In this case, all procedures performed in studies involving human participants were in accordance with the ethical standards of the institutional and/or national research committee(s) and with the Helsinki Declaration (as revised in 2013). Written informed consent was obtained from the patient for publication of this study and any accompanying images.

\section{Discussion}

There are 4 major LBCV variants: (I) alterations of the anatomical sites [e.g., the coronary sinus (1) and left atrium (2)] into which blood from the LBCV converges; (II) abnormal LBCV courses [e.g., inferior or posterior aortic arch course (3-5), intrathymic course (6), and 


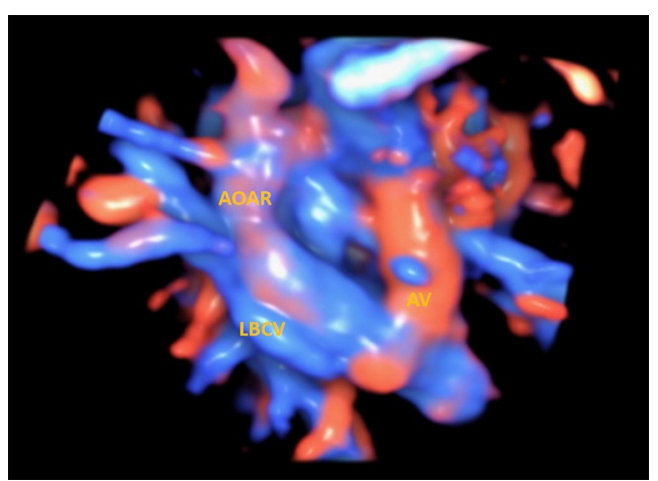

Figure 4 STIC volumetric imaging shows the LBCV traveling to the AV posterior to the DAO. STIC, spatio-temporal image correlation; LBCV, left brachiocephalic vein; AV, azygos vein; DAO, descending aorta.

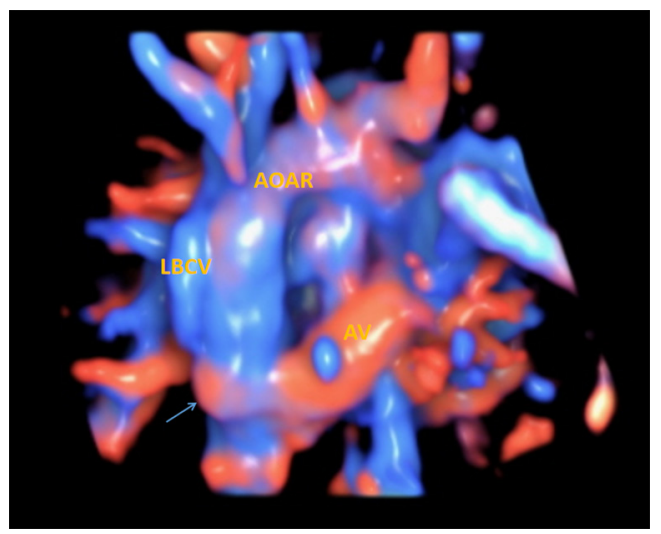

Figure 5 STIC volumetric imaging shows the LBCV traveling posterior to the DAO (arrow) and merging into the SVC via the AV. STIC, spatio-temporal image correlation; DAO, descending aorta; SVC, superior vena cava; AV, azygos vein.

retrotracheal or retroesophageal course $(7,8)]$; (III) duplication of the $\operatorname{LBCV}(9,10)$; and (IV) shunts between the LBCV and communicating branch of the other vein (e.g., the pulmonary vein) (11).

First reported by Yigit et al. (7), retroesophageal or retrotracheal LBCV is extremely rare. The incidence is unknown and only a few cases have been reported so far. According to literature, this $\mathrm{LBCV}$ variant travels posterior to the trachea or esophagus and then merges into the superior vena cava via the arch of the azygos vein. When the embryo is $3-4 \mathrm{~mm}$ long, 3 pairs of venous inputs (the vitelline vein, umbilical vein, and common cardinal vein) appear from the caudal side of the venous sinus. The common cardinal vein is rapidly divided into the anterior and posterior cardinal veins. The anterior cardinal vein extends along the cephalic side of the anterior intestine on both sides to drain blood from the head veins. At about the eighth gestational week, an anastomosing branch of the thymus-thyroid vein network arises between the right and left anterior cardinal veins and later develops into the LBCV. The portion of the right anterior cardinal vein located between the subclavian vein and the LBCV develops into the right brachiocephalic vein, while its caudal portion and the right common cardinal vein develop into the superior vena cava. The specific mechanism is unknown, If the anastomosing branch of the thymus-thyroid vein network is not formed, the LBCV may merge into the azygous vein or hemiazygos vein before merging into the superior vena cava (7); a retroesophageal or retrotracheal course may develop.

In recent years, the increasingly optimized resolution of obstetric ultrasound equipment has enabled he clear visualization of the vascular courses in the neck of fetuses. When the LBCV was routinely scanned by ultrasound, through the cross-sectional view of the bilateral subclavian arteries typically, the normal fetus can show that a straight course of the LBCV from the left neck to the right superior vena cava. During the dynamic ultrasound scanning of left cervical and thoracic areas, the normal fetus can show that the left subclavian vein and the left internal jugular vein converging to form he LBCV, which travels downwards to the right side and forms the superior vena cava together with the right brachiocephalic vein above the aortic arch. In our current case, the $3 \mathrm{VV}$ of the fetus showed an abnormal vessel visible on the left side of the pulmonary artery. Pulsed-wave Doppler ultrasound showed a venous spectrum. Dynamic tracing of this vein showed that it was formed by the confluence of the left internal jugular vein and the left subclavian vein. The possibility of LBCV was considered. The vein traveled downward posterior to the trachea and joined the superior vena cava through the azygous vein. Spatio-temporal image correlation (STIC) was then used to reconstruct the fetal large vessels in 3-dimensional (3D), which again showed that the left internal jugular vein and the left subclavian vein converged to form the $\mathrm{LBCV}$, which then traveled posterior to the trachea and joined the superior vena cava via the azygous vein. As a $3 \mathrm{D}$ technique, STIC can visualize the abnormal courses of LBCV, thus improving the diagnostic accuracy. Prenatal examination can also be performed by fetal cardiac MRI. It was found that a retrotracheal $\mathrm{LBCV}$ passing 
posterior to the trachea, joining the azygos vein, and finally draining into the superior vena cava (12).

The diameter of LBCV increased with gestational age, a report of 431 cases of normal fetuses were measured, and the diameter of LBCV was summed up as a calculation equation $(13)$ : diameter $=(0.1442 \times \mathrm{GA})-0.8812$, where diameter is measured in $\mathrm{mm}$ and GA is gestational age in weeks. At 23 weeks, the diameter of the LBCV was about $2.4 \mathrm{~mm}$. The diameter of the $\mathrm{LBCV}$ in this case was in the normal range.

As the $3 \mathrm{VV}$ provides a transverse view of the vein on the left side of the pulmonary artery, this sign needs to be differentiated from other diseases such as total anomalous pulmonary venous return (supracardiac type) and persistent left superior vena cava. Supracardiac anomalous pulmonary venous return is a condition in which some or all of the pulmonary veins fail to connect to the left atrium; rather, they merge into a vertical vein that later connects to the right atrium or other reflux vessels. This vertical vein may be located to the left of the pulmonary artery and can be identified by tracing the pulmonary veins. The permanent left superior vena cava, formed by incomplete degeneration of the proximal left anterior cardinal vein, can be divided into type I (the permanent left superior vena cava connecting to the coronary sinus, which is more common) and type II (the permanent left superior vena cava connecting to the left atrium, which is less common). It can be identified by tracing the course of the left superior vena cava.

Retroesophageal or retrotracheal LBCV usually has a good prognosis. Very few children with LBCV have symptoms similar to those of aberrant right subclavian artery: they are typically asymptomatic, with occasional respiratory symptoms or dysphagia (7). However, the presence of LBCV may affect the approach of a cardiac or thoracic surgery. Surgeons should have a detailed and adequate understanding of LBCV variants before surgery and choose the appropriate surgical path.

In conclusion, in this article we summarize the $2 \mathrm{D}$, color Doppler, and STIC findings of an isolated retrotracheal LBCV, which and may inform the sonographic diagnosis of such variants. However, as the child is still young and had no obvious clinical symptoms, a long-term follow-up is warranted. When an abnormal vascular cross-section is found on the left side of the pulmonary artery on the $3 \mathrm{VV}$, the diagnosis of LBCV may be made after tracing its superior and inferior vascular compositions and course by using 2D, color Doppler, and STIC techniques.

\section{Acknowledgments}

Funding: None.

\section{Footnote}

Reporting Checklist: The authors have completed the CARE reporting checklist. Available at https://dx.doi. org/10.21037/tp-21-230

Conflicts of Interest: All authors have completed the ICMJE uniform disclosure form (available at https://dx.doi. org/10.21037/tp-21-230). The authors have no conflicts of interest to declare.

Etbical Statement: The authors are accountable for all aspects of the work in ensuring that questions related to the accuracy or integrity of any part of the work are appropriately investigated and resolved. Written informed consent was obtained from the patient for publication of this study and any accompanying images. All procedures performed in studies involving human participants were in accordance with the ethical standards of the institutional and/or national research committee(s) and with the Helsinki Declaration (as revised in 2013).

Open Access Statement: This is an Open Access article distributed in accordance with the Creative Commons Attribution-NonCommercial-NoDerivs 4.0 International License (CC BY-NC-ND 4.0), which permits the noncommercial replication and distribution of the article with the strict proviso that no changes or edits are made and the original work is properly cited (including links to both the formal publication through the relevant DOI and the license). See: https://creativecommons.org/licenses/by-nc$\mathrm{nd} / 4.0 /$.

\section{References}

1. Singh B, Ramsaroop L, Maharaj J, et al. Case of double superior vena cava. Clin Anat 2005;18:366-9.

2. Townsend MD, Jonas RA, Moskowitz WB. Congenital communication of a retroaortic innominate vein with both the left and right atria in the presence of a normal coronary sinus. Pediatr Cardiol 2008;29:823-6.

3. Kulkarni S, Jain S, Kasar P, et al. Retroaortic left innominate vein - Incidence, association with congenital heart defects, embryology, and clinical significance. Ann 
Pediatr Cardiol 2008;1:139-41.

4. Semionov A, Kosiuk J. Incidental retroaortic left innominate vein in adult patient. Radiol Case Rep 2017;12:475-8.

5. Chen SJ, Liu KL, Chen HY, et al. Anomalous brachiocephalic vein: CT, embryology, and clinical implications. AJR Am J Roentgenol 2005;184:1235-40.

6. Karl K, Sinkovskaya E, Abuhamad A, et al. Intrathymic and other anomalous courses of the left brachiocephalic vein in the fetus. Ultrasound Obstet Gynecol 2016;48:464-9.

7. Yigit AE, Haliloglu M, Karcaaltincaba M, et al. Retrotracheal aberrant left brachiocephalic vein: CT findings. Pediatr Radiol 2008;38:322-4.

8. Ming Z, Aimin S, Rui H. Evaluation of the anomalous retroesophageal left brachiocephalic vein in Chinese children using multidetector CT. Pediatr Radiol 2009;39:343-7.

Cite this article as: Zhao Y, Ji X, Ding L, Xia Y, Wu Y, Xu $\mathrm{R}, \mathrm{Wu}$ N. Diagnosis of retrotracheal left brachiocephalic vein with prenatal ultrasound: a case report. Transl Pediatr 2021;10(7):1960-1964. doi: 10.21037/tp-21-230
9. Kawamura I, Hojo R, Fukamizu S. A case of pacemaker implantation in the patient with duplication of the left innominate vein: a case report. Springerplus 2016;5:515.

10. Malakan Rad E. Circumaortic double left innominate vein: Two novel echocardiographic diagnostic clues for a tricky diagnosis. Echocardiography 2018;35:1898-900.

11. Yoshibayashi M, Arakaki Y, Yamada O, et al. Univentricular heart and pulmonary stenosis with a right-to-left shunt between the innominate and pulmonary veins after Glenn operation: a case report. J Cardiol 1987;17:199-206.

12. Dong SZ, Zhu M. MR imaging of subaortic and retroesophageal anomalous courses of the left brachiocephalic vein in the fetus. Sci Rep 2018;8:14781.

13. Sinkovskaya E, Abuhamad A, Horton S, et al. Fetal left brachiocephalic vein in normal and abnormal conditions. Ultrasound Obstet Gynecol 2012;40:542-8.

(English Language Editor: J. Gray) 